\title{
Histochemical investigation into the molecular mechanisms of malignant transformation in a benign glomus tumour
}

\author{
L Hegyi, G C Cormack, J W Grant
}

\begin{abstract}
A glomangiosarcoma arose in a benign glomus tumour. The histological and immunohistochemical characteristics of the tumour were investigated. Apoptotic cells were identified by terminal deoxynucleotidyl transferase (TdT) mediated dUTP-biotin nick end labelling (TUNEL). The proportion of apoptotic cells was found to be low and TUNEL positive nuclei were present in the benign part of the tumour. Bcl-2 protein, an inhibitor of apoptosis, was strongly expressed in the glomangiosarcoma with only weak staining in the benign area. The proliferation index of the glomangiosarcoma was almost 10-fold higher than that of the benign glomus tumour. Numerous nuclei in the glomangiosarcoma were intensely stained for the tumour suppressor protein p53. The results of the this study may contribute to an understanding of the molecular basis of malignant transformation in benign glomus tumours. (f Clin Pathol 1998;51:872-874)
\end{abstract}

Keywords: glomangiosarcoma; glomus tumour; apoptosis

Glomus tumours are uncommon benign neoplasms causing paroxysmal pain. ${ }^{1}$ They are composed of modified, epithelioid smooth muscle cells arising from the glomus body. ${ }^{12}$ Malignant forms of glomus tumour are exceptionally rare. ${ }^{2-5}$ We describe a case of glomangiosarcoma arising in a benign glomus tumour. We investigated the biology of the tumour cells, including the molecular mechanisms of malignant transformation, using immunohistochemical methods.

Hospital, Hills Road, Cambridge, UK:

Department of Plastic

Surgery

G C Cormack

Department of Histopathology

J W Grant

Correspondence to: Dr Laszlo Hegyi,

Department of Medicine, University of Cambridge, Addenbrooke's Hospital,

Level 5, Hills Road,

Cambridge CB2 2QQ, UK; email:

lhegyi@mole.bio.cam.ac.uk

Accepted for publication 17 June 1998
Three months later a small tender swelling recurred on the scar. This was excised. There were no surgical complications.
Methods

Formalin fixed, paraffin embedded sections in $0.01 \mathrm{~mol} /$ litre sodium citrate buffer at $\mathrm{pH} 6$ were treated in a microwave oven for 10 minutes. They were then incubated with monoclonal antibodies (Dako, unless otherwise stated) to desmin, vimentin, skeletal myosin (Sigma), $\alpha$-smooth muscle actin (Sigma), human endothelial cells (CD34, QBend10; Novocastra), factor VIII related antigen, Ki-67, p53 (mutant and wild type), and Bcl-2. Subsequently immunohistochemical staining was completed using the avidinbiotin complex (ABC) method.

Terminal deoxynucleotidyl transferase (TdT) mediated dUTP-biotin nick end labelling (TUNEL) was performed essentially as described before. ${ }^{6}$ Briefly, the sections were incubated with $\mathrm{TdT}(0.1 \mathrm{U} / \mu \mathrm{l})$ and biotinylated dUTP (Boehringer Mannheim) for 30 minutes at $37^{\circ} \mathrm{C}$. Binding sites of biotinylated dUTP were visualised using the ABC method.

To quantify the results at least 10 randomly selected, non-overlapping fields were counted in each zone of the tumour. The field was defined by a $0.5 \mathrm{~cm}$ square grid with $0.5 \mathrm{~mm}$ divisions, using a $\times 40$ objective and $\times 10$ eyepiece. At least 3000 cells were examined in every area, and staining was carried out on at least two sections. A proliferation index was calculated from the formula: (number of $\mathrm{Ki}-67$ positive nuclei/total nuclei) $\times 100$. Similarly, an apoptotic index was determined from the formula: (number of TUNEL positive nuclei/ total nuclei) $\times 100$.

Results

In the dermis the $7 \times 5 \mathrm{~mm}$ recurrent tumour was enclosed by a fibrous pseudocapsule and consisted of two distinct components (fig 1). One was a crescent at the lateral and deep margins of the lesion and comprised thin walled vascular spaces of varying size which were surrounded by relatively uniform cells having round to oval nuclei with pale staining eosinophilic cytoplasm. The solid sheets of cells were embedded in a stroma which focally showed myxoid change. The PAS reaction revealed the characteristic "chicken wire" network of basal lamina. ${ }^{2}$ The appearances of this component were those of a benign glomus tumour and resembled those seen in the first biopsy described above.

A second component, the bulk of the lesion and within the pseudocapsule, consisted of spindle shaped and ovoid cells, chiefly in a fascicular arrangement forming whorl-like struc- 


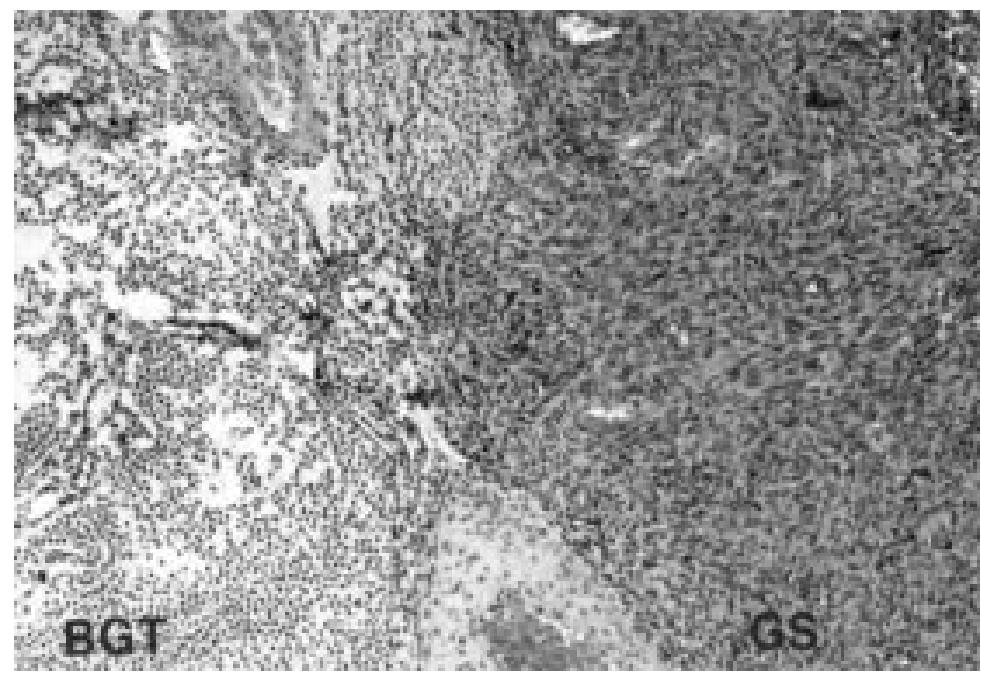

Figure 1 Interface of benign glomus tumour and glomangiosarcoma (haematoxylin and eosin).

tures (fig 1). The nuclei of these cells showed hyperchromasia and most had a single prominent nucleolus. There were scattered mitotic figures (fig 2), unlike the histologically benign part of the tumour. There was no evidence of infiltration of the adjacent connective tissue.

Both components of the tumour expressed vimentin and smooth muscle actin. The intensity of vimentin staining appeared to be the same in both parts, but actin showed more intense staining in the benign zone than in the glomangiosarcoma. Neither component of the tumour showed staining for myosin, desmin, QBend10, and factor VIII related antigen. Although Ki-67 positive nuclei were found in both the benign and the sarcomatous components, the numbers of cells having these nuclei were apparently different in the two zones. The proliferation index was $0.15 \%$ in the benign glomus tumour but almost 10 times higher in the glomangiosarcoma, at $1.13 \%$.

The finding of nuclear staining by TUNEL in the benign glomus tumour suggested that these cells were apoptotic. ${ }^{6}$ The apoptotic index in the benign glomus tumour was $0.05 \%$.

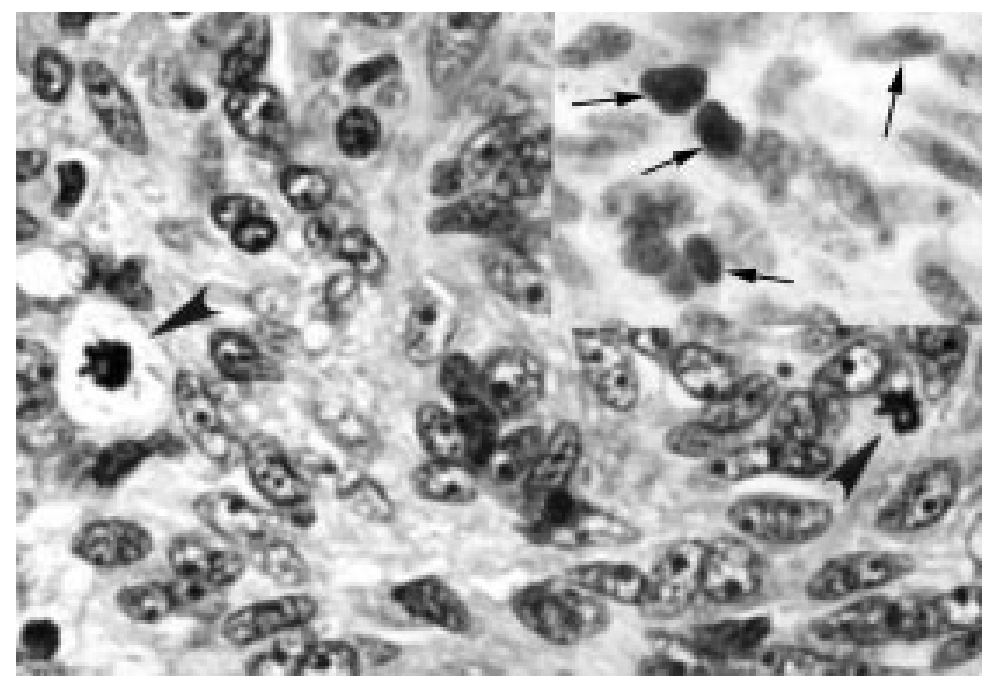

Figure 2 Higher magnification of glomangiosarcoma showing atypical tumour cells with polymorphic nuclei and mitoses (arrowheads) (haematoxylin and eosin). An adjacent section shows p53 stained nuclei (arrows) (inset).
There were no TUNEL labelled cells in the sections of glomangiosarcoma examined. There was no necrosis in either the benign or the sarcomatous components.

Both parts of the tumour expressed Bcl-2 protein but the staining appeared to be more intense in the glomangiosarcoma than in the benign glomus tumour. Weak expression of p53 protein was seen in a few cells of the benign glomus tumour but many nuclei were intensely stained in the glomangiosarcoma (fig 2). The bulk of p53 positive nuclei were in the zone of glomangiosarcoma and there were no intensely staining nuclei in the benign glomus tumour.

\section{Discussion}

Malignant glomus tumours have been subdivided into several categories based on clinicopathological criteria. Glomangiosarcoma arising in a benign glomus tumour has been distinguished from locally infiltrative glomus tumour and de novo glomangiosarcoma. ${ }^{4}$ Most of the reported cases represented examples of the former group, in which a sarcomatous tumour arose in a benign glomus tumour. ${ }^{2-5}$

The ultrastructural and certain immunohistochemical features of malignant glomus tumours have been described. ${ }^{3-5}$ It has been shown that the tumour cells of the sarcomatous zone share many common characteristics with those of the benign glomus tumour. ${ }^{3}$

The results of our study are in accord with those of the previous papers. ${ }^{2-5}$ We found that the cells of both the glomangiosarcoma and benign glomus tumour stained for vimentin and actin. Likewise in the tumour reported by Aiba et $a l,{ }^{3}$ actin was stained more intensely in the zone of benign glomus tumour than in the glomangiosarcoma, but there was no apparent difference in the intensity of vimentin staining between the two parts of the tumour described here.

In the present study some cells of both the benign glomus tumour and the glomangiosarcoma areas were stained for $\mathrm{Ki}-67$, which is a nuclear antigen expressed during all phases of the cell cycle but not in non-cycling (G0 phase) cells. ${ }^{7}$ The low proliferation index in the benign glomus tumour suggests that this is a slowly growing tumour. However, the effective rate of tumour growth in this zone was less because of the occurrence of apoptosis. In the glomangiosarcoma the proliferation index was almost 10-fold higher than in the benign glomus tumour, with virtually absent apoptosis. The possibility that there may be minimal numbers of apoptotic cells in the glomangiosarcoma cannot entirely be excluded but more tissue than was available would be required to determine the precise apoptotic index in this region.

In this study we have shown that glomangiosarcoma is more intensely stained for $\mathrm{Bcl}-2$ protein than benign glomus tumour. $\mathrm{Bcl}-2$, which is localised to subcellular compartments including nuclear and mitochondrial membrane, ${ }^{8}$ blocks apoptotic death in vitro. ${ }^{8}$ The overexpression of $\mathrm{Bcl}-2$ in the glomangiosarcoma may repress the deletion of sarcoma cells by apoptosis, which is in accordance with 
the findings obtained by the TUNEL staining showing the absence of apoptotic cells in the sections of glomangiosarcoma.

Immunohistochemistry revealed intense nuclear staining for the tumour suppressor protein p53 in glomangiosarcoma cells of the present tumour. This finding is consistent with the results of Watanabe et al. ${ }^{5}$ The faint nuclear staining in the zone of benign glomus tumour might be a result of the vigorous method of antigen retrieval used in our study.

p53 Protein has antiproliferative effects, including growth arrest and a role in induction of apoptosis. ${ }^{9}$ Its function is also essential in repair following DNA damage. ${ }^{9}$ The loss of normal p53 function is associated with cell transformation in vitro and the development of neoplasms in vivo. ${ }^{9}{ }^{10}$ The inactivation of wildtype p53 could be caused by sequestration or destruction by viral oncoproteins,,${ }^{9} 10$ binding to cellular gene products such as murine double minute-2 (mdm2) protein, ${ }^{10}$ or mutations of the p53 gene. ${ }^{9} 10$

The concentration of $\mathrm{p} 53$, which is an unstable protein, is low in normal cells and is often undetectable. ${ }^{10}$ The intense nuclear staining for p53 in the glomangiosarcoma, but not in the benign glomus tumour, suggests that the inactivation of $\mathrm{p} 53$ protein is involved in the malignant transformation of benign glomus tumour. The reasons for this are unknown but the loss of normal p53 function might contribute to impaired DNA repair, ${ }^{9}$ decreased apoptosis, and the development of malignant tumour in the benign glomus tumour.

Further investigation of the exceptionally rare phenomenon of glomangiosarcoma arising in a benign glomus tumour is required.

We are grateful to Drs D G D Wight, M J Mitchinson, and J N Skepper for advice. We also thank Miss S Howard for her technical support and $\mathrm{Mr} \mathrm{C}$ Burton for his photographic assistance.

1 Masson P. Le glomus neuromyoartériel des régions tactiles et ses tumeurs. Lyon Chir 1924;21:257-80.

2 Enzinger FM, Weiss SW. Perivascular tumors. In: Soft tissue tumors. St Louis: CV Mosby, 1995:701-33.

3 Aiba M, Hirayama A, Kuramochi S. Glomangiosarcoma in a glomus tumor. Cancer 1988;61:1467-71.

4 Gould EW, Manivel JC, Albores-Saavedra J, et al. Locally Gould EW, Manivel JC, Albores-Saavedra J, et al. Locally
infiltrative glomus tumors and glomangiosarcomas. Cancer infiltrative glomus
1990;65:310-18.

5 Watanabe K, Hoshi N, Tsu-Ura Y, et al. A case of glomangiosarcoma. Fukushima f Med Sci 1995;41:71-7.

6 Hegyi L, Skepper JN, Cary NRB, et al. Foam cell apoptosis and the development of the lipid core of human atherosclerosis. F Pathol 1996;180:423-9.

7 Verheijen R, Kuijpers HJ, van Driel R, et al. Ki-67 detects a nuclear matrix-associated proliferation-related antigen. II. Localization in mitotic cells and association with chromosomes. F Cell Sci 1989;92:531-40.

8 Hockenbery D, Nunez G, Milliman C, et al. Bcl-2 is an inner mitochondrial membrane protein that blocks programmed cell death. Nature 1990;348:334-6.

9 Gottlieb TM, Oren M. p53 in growth control and neoplasia. Biochim Biophys Acta 1996;1287:77-102.

10 Chang F, Syrjänen S, Tervahauta A, et al. Tumourigenesis associated with the p53 tumour suppressor gene. Br f Cancer 1993;68:653-61. 